\title{
Extended focus depth for Fourier domain optical coherence microscopy
}

\author{
R. A. Leitgeb, M. Villiger, A. H. Bachmann, L. Steinmann, and T. Lasser \\ Laboratoire d'Optique Biomedicale, Ecole Polytechnique Federale de Lausanne, CH-1015 Lausanne, Switzerland \\ Center of Biomedical Engineering and Physics, Medical University of Vienna, Waehringerstrasse 13, \\ A-1090 Vienna, Austria \\ Received April 4, 2006; revised May 24, 2006; accepted May 29, 2006; \\ posted June 1, 2006 (Doc. ID 69650); published July 25, 2006 \\ We report on a new detection scheme for Fourier domain optical coherence microscopy that exhibits high \\ transverse resolution along an axially extended focal range. Nearly constant transverse resolution of \\ $\sim 1.5 \mu \mathrm{m}$ along a focal range of $200 \mu \mathrm{m}$ is experimentally verified with a maximum sensitivity of $105 \mathrm{~dB}$. A \\ broad-bandwidth Ti:sapphire laser allowed for an axial resolution of $3 \mu \mathrm{m}$ in air. (C) 2006 Optical Society of \\ America \\ OCIS codes: $110.4500,170.6900,170.3880$.
}

Fourier domain optical coherence tomography (FDOCT) combines high-speed imaging with high sensitivity and axial resolution. ${ }^{1-3}$ The reason for this is an efficient detection scheme that allows acquisition of a full depth profile synchronously without an axial scan. In FDOCT the axial sample structure is encoded in the spectral interference pattern recorded via a spectrometer at the exit of an interferometer. The sample reconstruction is obtained via inverse Fourier transform of such a pattern. Hence the imaging speed is essentially limited by the frame rate of the array detector used. In contrast with other optical imaging methods, the depth resolution $\delta z$ is independent of the numerical detection aperture (NA). The depth resolution is given by the coherence length of the light source employed, which is proportional to $\lambda_{C}^{2} / \Delta \lambda$, with $\lambda_{C}$ being the center wavelength and $\Delta \lambda$ the spectral bandwidth. The lateral resolution, on the other hand, is still determined by the focusing objective: if the objective entrance pupil is larger than the Gaussian beam waist diameter $d$ at this position, we can use the $1 / e^{2}$-intensity beam waist radius in the focal distance $f$ to define the lateral resolution as $\delta r_{G}=\lambda_{C} /(\pi d / 2 f) \approx \lambda_{C} /(\pi \mathrm{NA})$. Since FDOCT acquires the whole axial depth structure in parallel, it is important to consider the actual focal range $\Delta z$, i.e., the range at which the transverse beam extent remains within predefined borders. For Gaussian beams one uses the Rayleigh range criterion defining the focal range as twice the distance at which the $1 / e^{2}$ beam waist increased by a factor of $\sqrt{2}: \Delta z_{G}=8 \lambda_{C}(f / d)^{2} / \pi$ $\propto 1 / \mathrm{NA}^{2}$. It is evident that large NAs lead to fast degradation of transverse resolution since the focal range shortens with the square of the NA. This dilemma has strong implications for applying FDOCT, with all its potential advantages such as imaging speed, detection sensitivity, and the availability of signal phase, to the field of microscopy, i.e., Fourier domain optical coherence microscopy ${ }^{4}$ (FDOCM). Therefore different approaches have been followed to maintain high transverse resolution along an effectively increased focal range. The strategies either improve the optical setup or involve elaborate deconvolution algorithms in postprocessing. ${ }^{5-7}$ The latter depend critically on signal-to-noise ratio (SNR), which is problematic for inherently small signals outside the confocal range. The first optical solutions to increase the focal range of time domain OCT systems have been dynamic focusing schemes. ${ }^{8,9}$ Even though C-mode scanning has been adapted for FDOCM detection, dynamic focusing techniques in general sacrifice imaging speed. To maintain the speed advantage of FDOCM it is necessary to look for true extended focus schemes. A first attempt, again for time domain OCT, has been using an axicon lens in front of the sample instead of an objective. ${ }^{10}$ Conical lenses give rise to Bessel beam field distribution in the focal plane, whereas in the far field they create a ring shape. The important advantage is that the central lobe extends along the full cross-sectional volume with a constant transverse extension. Nevertheless, the central lobe of a Bessel field carries only $\sim 5 \%$ of the total power, compared with $86 \%$ of a Gaussian field distribution that is localized within the $1 / e^{2}$ beam radius. This difference becomes even more dramatic if the detection is performed along the reverse path through the axicon, ultimately limiting the application for biomedical samples.

In the present work we introduce a scheme that profits from the enhanced resolution performance of Bessel beam illumination but uses more efficient detection. For this task we take advantage of the ringshaped intensity distribution generated by an axicon lens in the far field: when this field is focused into the back focal plane of a microscope objective, the intensity distribution at the sample position after the objective is a Bessel field exhibiting an extended focal range. Decoupling the detection and illumination paths avoids the need for an intensity-critical double pass through the axicon lens. The detection will again be confocal, resulting in higher detection sensitivity. The Gaussian weighting of the Bessel field due to the confocal detection has the drawback of reducing the extended focal range, but the transverse resolution remains constant along the fully extended focal range. There is no exact analytic solution describing the intensity distribution after an axicon lens. Herman and Wiggins ${ }^{11}$ gave a solution for the intensity along the cross-sectional volume close to the 
optical axis as $I(\rho, z) \propto k \rho_{\alpha} \sin \alpha /\left(1-\sin ^{2} \alpha\right) J_{0}{ }^{2}(k \rho$ $\times \sin \alpha$ ), where $\rho$ is the radius perpendicular to the optical axis, $\rho_{\alpha}=z \tan \alpha$, $\sin \alpha$ is the effective NA of the illumination, $k$ is the center wavenumber of the light source, and $J_{0}$ is the zero-order Bessel function. The transverse resolution $\delta r_{B}$ is taken to be equal to the first zero of $J_{0}$ as $\delta r_{B}=1.202 \lambda_{C} /(\pi \mathrm{NA})$, which can be compared with the $1 / e^{2}$ Gaussian beam waist, $\delta r_{G}$. The axial cross-sectional range that defines the illumination depth is given by $\Delta z_{B}=2 \rho_{R} f / d \approx \rho_{R} / \mathrm{NA}$, with $\rho_{R}$ being the ring thickness and $d$ being the ring diameter at the image-side principal plane of the microscope objective [Fig. 1(a)].

The measurement setup is shown in Fig. 1: Light from a broad-bandwidth source (Ti:sapphire laser, Femtolasers, Inc.) with the center wavelength at $800 \mathrm{~nm}$ and a spectral bandwidth of $135 \mathrm{~nm}$ is split by a 50:50 fiber coupler into separate paths for the reference and sample arms. In the sample arm the collimated beam passes an axicon lens (Del Mar Photonics, $170^{\circ}$ apex angle) and is focused via achromat L2 $(f=100 \mathrm{~mm})$ into a ring of $d=8 \mathrm{~mm}$ diameter at the back focal plane of lens L3 (10× Zeiss Plan Neofluar). The ring width at the image-side principal plane of L3 is $\rho_{R}=130 \mu \mathrm{m}$, resulting in $\Delta z_{B}=560 \mu \mathrm{m}$. The effective NA of the sample illumination after L3 is 0.23 , resulting in a theoretical transverse resolution of $1.33 \mu \mathrm{m}$ over the full depth range $\Delta z_{B}$. The axial resolution is $3 \mu \mathrm{m}$ in air. The chromatic error due to the axicon dispersion is negligible since the apex angle of the axicon is large. The wavelengthdependent refraction angle after the axicon varies by only $0.014^{\circ}$ over the full bandwidth of $135 \mathrm{~nm}$, corresponding to a relative error of $0.53 \%$. The combination of axicon and lens L2 (dashed box) can be removed to realize a confocal (cf) FDOCM setup with an effective illumination NA of 0.12 . The backscattered light from the sample is diverted via beam splitter BS to the detection path, where lens L4 (10 $\times$ Zeiss Plan Neofluar) couples the light into a singlemode fiber with $\mathrm{NA}=0.12$. In the reference arm a block of BK7 glass serves as a dispersion balance, and the diaphragm is used to adjust the referencearm intensity for maximum sensitivity. ${ }^{2}$ The light is recombined via a 90:10 fiber coupler with polarization control paddles to maximize the fringe visibility of the spectral interference pattern. The latter is recorded by a spectrometer consisting of a transmission grating (Wasatch, Inc., 1200 lines/mm), a camera objective $(f=135 \mathrm{~mm})$, and a line detector (Atmel Aviiva) with 2048 pixels set to a frame rate of $5 \mathrm{kHz}$. The achieved spectrometer resolution is $\delta \lambda=0.08 \mathrm{~nm}$, allowing for a maximal system depth range of $1.97 \mathrm{~mm}$ in air. The sample is mounted on an $x-y$ translation stage with continuous $x$-scanning of $0.5 \mathrm{~mm} / \mathrm{s}$ during signal acquisition. Note that measurement of sensitivity and verification of the resolution with a resolution test target are difficult since we have dark field illumination of the sample [Fig. 1(a)]. On the other hand, the dark field scheme discards strong reflexes, e.g., of microscope coverslides, that otherwise might saturate the spectrometer sen-

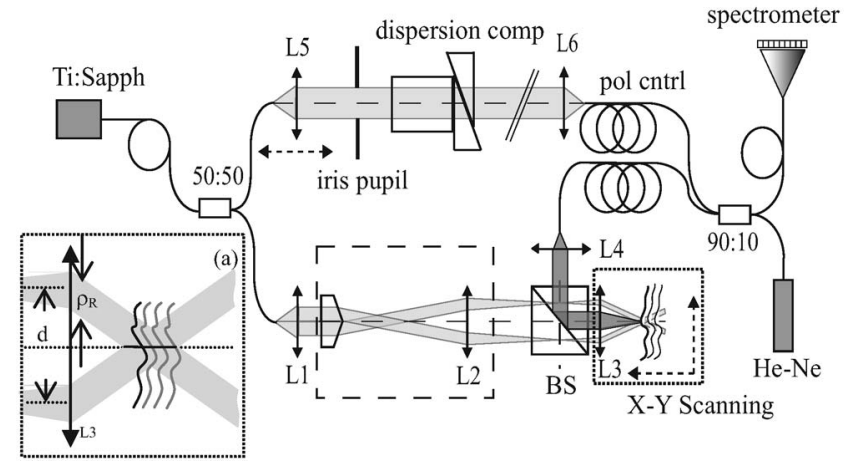

Fig. 1. Optical setup: L1-L6, lenses; BS, nonpolarizing beam splitter 50:50; He-Ne, helium-neon laser for adjustment; pol cntrl, polarization control. (a) Dark field illumination (light gray) and detection field (dark gray) at the sample position.

sor. The sensitivity of the cf FDOCM configuration is measured to be $112 \mathrm{~dB}$ with a power of $5 \mathrm{~mW}$ at a mirror sample in the focal plane of L3. An estimate for the Bessel beam setup sensitivity of $\sim 105 \mathrm{~dB}$ is readily obtained by comparison of SNR obtained for the same system parameters and sample. The FDOCT-intrinsic signal roll-off in both cases was $-7 \mathrm{~dB} / \mathrm{mm}$.

To test the resolution performance of extended field (xf) FDOCM we dispersed microbeads of $1 \mu \mathrm{m}$ diameter in Agar-Agar gel (0.25 vol. \%). The resulting tomograms for cf-FDOCM in comparison with $\mathrm{xf}$ FDOCM are displayed in Figs. 2(a) and 2(b). They consist of $6000 \times 350$ pixels covering a range of $600 \mu \mathrm{m} \times 500 \mu \mathrm{m}$. For Fig. 2(c) signals of single microbeads were selected and aligned along the optical axis for both cases. At a given depth only those signals that have the largest transverse extent are chosen to ensure that the signals correspond to approximately central particle cross sections. This representation allows direct comparison of transverse resolution. The plotted lines in Fig. 2(c) show theoretical predictions using Gaussian optics and the formula for Bessel beam illumination that are in good agreement with the measurements. The calculations assume a refractive index of 1.33 . The visible extended focal range of $\sim 200 \mu \mathrm{m}$ is smaller than the full geometric illumination depth of $\Delta z_{B}=560 \mu \mathrm{m}$. This is due to the Gaussian detection envelope, which acts axially as well as laterally as apodization for the Bessel field distribution. The focal detection range of the xf-FDOCM configuration [solid lines in Fig. 2(c)] is visibly larger than that of the actual confocal setup [dashed lines in Fig. 2(c)]: The simulated confocal case with $\mathrm{NA}=0.23$, identical to the illumination NA of the xf-FDOCM setup, is indicated by the dotted lines in Fig. 2(c). The xf-FDOCM system exhibits approximately the same transverse resolution but with the advantage of increased focus depth. Nevertheless, the price to pay for the extended focus depth is reduced sensitivity at the focal plane. To theoretically estimate the sensitivity loss of the proposed xfFDOCM scheme compared with that of conventional cf-FDOCM, the product of the Bessel illumination field and the Gaussian detection field is calculated. 
The result is shown in Fig. 2(d) (left): it displays the relative SNR in the shot-noise limit between the xfFDOCM and the cf-FDOCM cases: $Q(z)$ $=\mathrm{SNR}_{\mathrm{xf}} / \mathrm{SNR}_{\mathrm{cf}}=I_{\mathrm{xf}}(z) / I_{\mathrm{cf}}(z) . I_{\mathrm{xf}}(z)$ and $I_{\mathrm{cf}}(z)$ are the respective sample field intensities from a single-point scatterer at axial position $z$ that are coupled back to the detector. For the SNR expression it is further assumed that the backscattered sample field intensity is much smaller than that of the reference field. ${ }^{2}$ Within the displayed axial range the relative SNR curves are approximately symmetric with respect to the focal position $z=0$. The theoretical SNR difference between the Bessel field to the realized confocal case with $\mathrm{NA}=0.12$ [dashed curve in Fig. 2(d), left] at the focal position is $-5 \mathrm{~dB}$, which is in close agreement with our measured value of $\sim-7 \mathrm{~dB}$. For NA $=0.23$ [dotted curve in Fig. 2(d), left] we observe at the focal position a strong SNR advantage of almost $20 \mathrm{~dB}$ for the confocal case. Nevertheless, this advantage is rapidly lost outside the corresponding short confocal range of $20 \mu \mathrm{m}$, resulting in a net SNR advantage for the shown extended focal range over $200 \mu \mathrm{m}$. The light solid curve in Fig. 2(d) demonstrates the SNR advantage of xf-FDOCM compared with the case of Bessel field illumination and detection (double pass) Figure 2(d) (right) displays simulated transverse cross sections through the detected sample field intensities for the different configurations. The confocal detection causes suppression of Bessel field sidelobes (light solid curve), but there is a trade-off between the amount of sidelobe suppression and extended focal range. The performance for imaging biological samples is demonstrated in Fig. 3, showing the three-dimensional cellular structure of
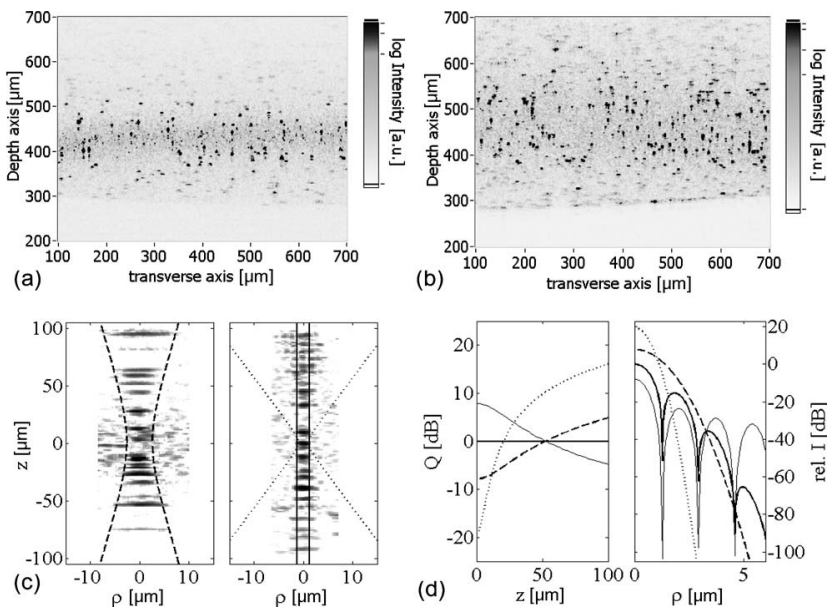

Fig. 2. Tomograms of $1 \mu \mathrm{m}$ beads for (a) cf-FDOCM, (b) xfFDOCM; (c) close-up of selected rearranged microbead signals with indicated theoretical resolution for cf-FDOCM with NA=0.12 (dashed line), NA=0.23 (dotted line), and xfFDOCM (solid line); (d) (left) $Q$-factor for $x f / c f, ~ N A=0.12$ (dashed curve), and NA=0.23 (dotted curve); $x f / x f$ (double pass) (light solid curve). (right) Transverse detected intensity profiles normalized to the xf-FDOCM intensity maximum for cf, $\mathrm{NA}=0.12$ (dashed curve), $\mathrm{NA}=0.23$ (dotted curve), xf (solid curve), xf (double pass) (light solid curve).

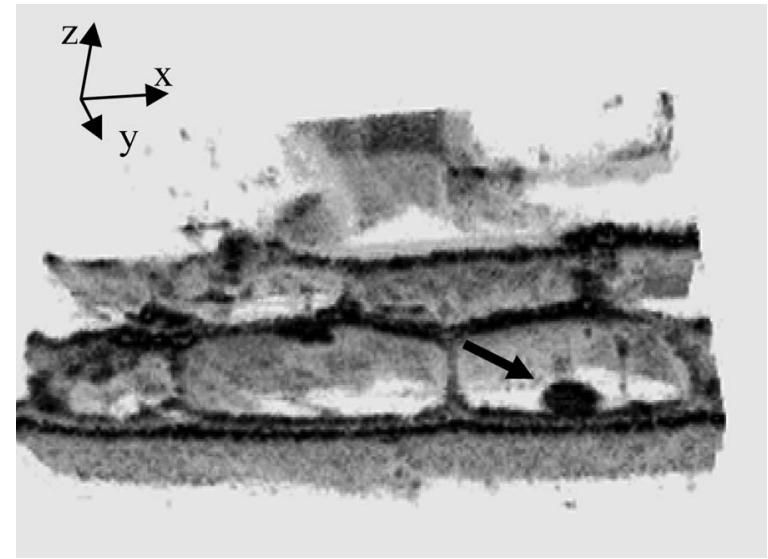

Fig. 3. Rendered three-dimensional image of onion skin cells [1024 $(x) \times 150(y) \times 105(z)$ voxels covering a geometric volume of $250 \mu \mathrm{m} \times 30 \mu \mathrm{m} \times 150 \mu \mathrm{m}]$. The arrow indicates a cell core.

an onion skin. The high transverse resolution allows resolution of subcellular details such as the indicated cell core.

In conclusion, we have presented the concept of $\mathrm{xf}-$ FDOCM, which allows increased focal depth ranging by employing Bessel beams. We obtain an almost tenfold gain in focal depth range with $\sim 1.5 \mu \mathrm{m}$ transverse resolution. Additionally, the proposed extended focus scheme has the flexibility to incorporate beam scanning devices.

This research was funded by the Swiss National Fonds (SNF grant 205321-10974). R. Leitgeb's e-mail address is rainerleitgeb@epfl.ch.

\section{References}

1. A. F. Fercher, C. K. Hitzenberger, G. Kamp, and S. Y. Elzaiat, Opt. Commun. 117, 43 (1995).

2. R. Leitgeb, C. K. Hitzenberger, and A. F. Fercher, Opt. Express 11, 889 (2003).

3. N. Nassif, B. Cense, B. H. Park, S. H. Yun, T. C. Chen, B. E. Bouma, G. J. Tearney, and J. F. de Boer, Opt. Lett. 29, 480 (2004).

4. M. A. Choma, A. K. Ellerbee, C. Yang, T. L. Creazzo, and J. A. Izatt, Opt. Lett. 30, 1162 (2005).

5. T. S. Ralston, D. L. Marks, F. Kamalabadi, and S. A. Boppart, IEEE Trans. Image Process. 14, 1254 (2005).

6. Y. Yasuno, J. I. Sugisaka, Y. Sando, Y. Nakamura, S. Makita, M. Itoh, and T. Yatagai, Opt. Express 14, 1006 (2006).

7. Y. Yasuno, Y. Sando, J. I. Sugisaka, T. Endo, S. Makita, G. Aoki, M. Itoh, and T. Yatagai, Opt. Quantum Electron. 37, 1185 (2005).

8. J. M. Schmitt, S. L. Lee, and K. M. Yung, Opt. Commun. 142, 203 (1997).

9. R. Huber, M. Wojtkowski, J. G. Fujimoto, J. Y. Jiang, and A. Cable, Opt. Express 13, 10523 (2006).

10. Z. H. Ding, H. W. Ren, Y. H. Zhao, J. S. Nelson, and Z. P. Chen, Opt. Lett. 27, 243 (2002).

11. R. M. Herman and T. A. Wiggins, J. Opt. Soc. Am. A 8, 932 (1991). 